\title{
BRAIN, SUBDURAL AND EPIDURAL ABSCESSES IN CHILDREN - 11 YEAR RETROSPECTIVE REVIEW
}

Ana Brett ${ }^{1,2}$, Inês Santos ${ }^{2}$, Fernanda Rodrigues ${ }^{1,2}$

${ }^{1}$ Hospital Pediátrico- Centro Hospitalar e Universitário de Coimbra, Serviço de Urgência e Unidade de Infecciologia, Coimbra, Portugal

2Universidade de Coimbra, Faculdade de Medicina, Coimbra, Portugal

\section{BACKGROUND AND AIMS}

Brain, subdural and epidural abscesses, although rare in the paediatric population, have significant morbidity and mortality. At present, there is a lack of evidence in the best approach regarding these patients, as guidelines are not consensual and the data published regarding these infections is scarce.

This study aimed to characterise children and adolescents with these abscesses and their medical-surgical approach over a period of 11 years.

\section{METHODS}

Retrospective descriptive study of the medical records of children and adolescents diagnosed with brain subdural or epidural abscesses at Hospital Pediátrico of Centro Hospitalar e Universitário de Coimbra during a period of 11 years (July $1^{\text {st }} 2006$ to June 30 ${ }^{\text {th }}$ 2017). Until February 2011 children from 0 to 13 years old could be admitted to hospital and after that from 0 to 18 years of age. Demographic data, comorbidities, predisposing factors, previous antibiotic treatment, clinical features, initial imaging, laboratorial results, medical-surgical treatment, time gap between relevant clinical landmarks, diagnosis and treatment, microbiology and postdischarge clinical follow-up were analysed.

\section{RESULTS}

Over 11 years, 16 cases of brain, subdural or epidural abscesses were diagnosed. Eleven (69\%) were in male patients and the median age of diagnosis was 8.8 years.

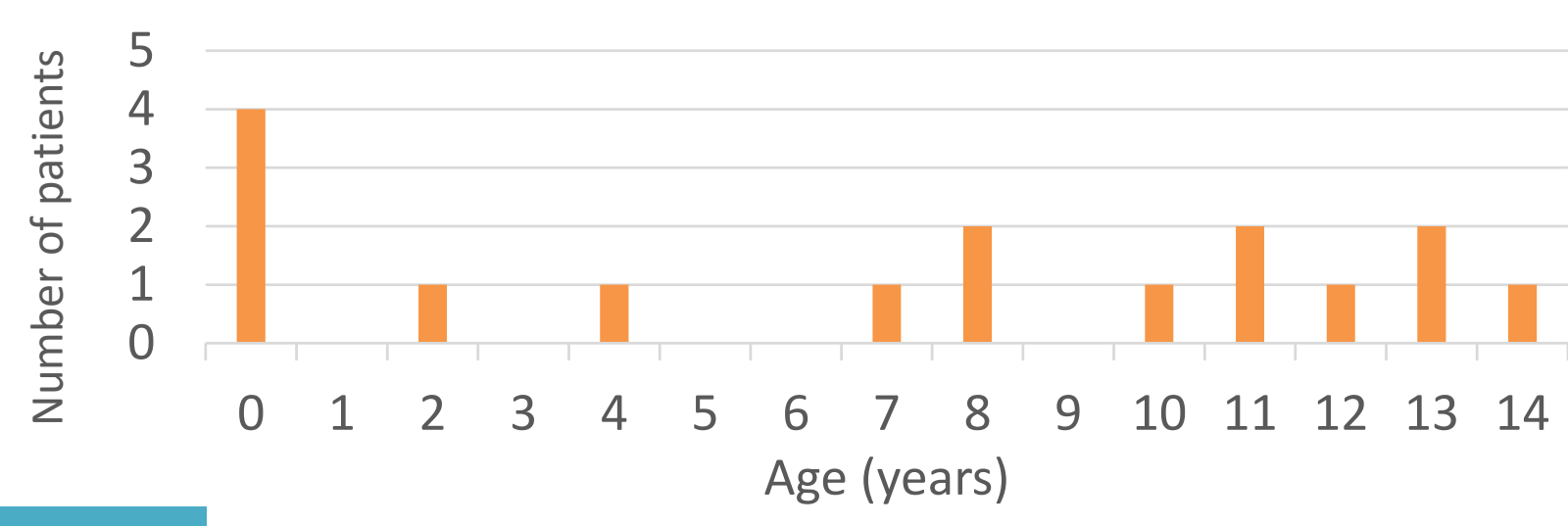

Predisposing factors

$-13(81,3 \%)$ were identified with predisposing factors, $2(12,5 \%)$ with two or more
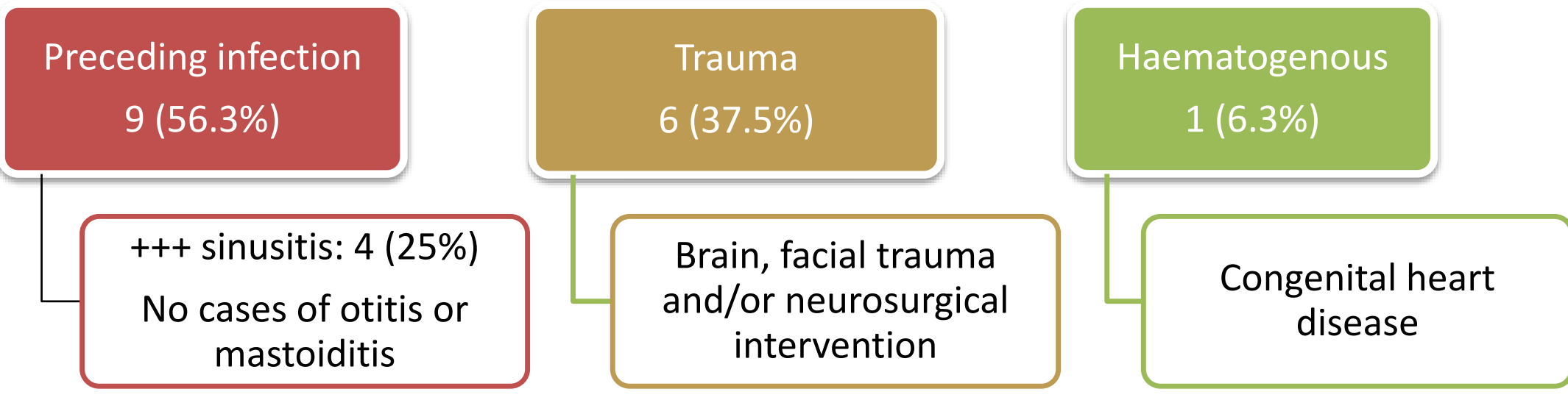

\section{Symptoms}

- Classic triad of headache, fever and focal neurological findings: 6 (37.5\%)

- Absence of neurological signs/symptoms: 4 (25\%)

- Non-specific symptoms predominated: fever, vomiting, constipation, cough, urinary tract retention, ...

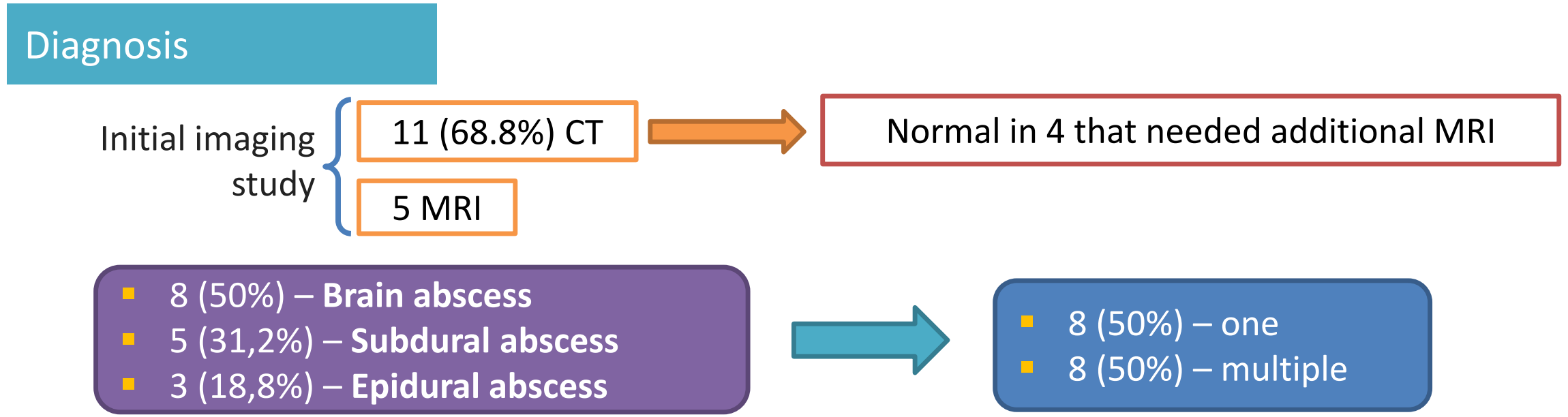

- Median time from initial symptoms to diagnosis: 10 days (0-55 days)

Microbiology

- 20 isolates, multiple bacteria in 2 cases

\begin{tabular}{|c|c|c|c|c|c|}
\hline \multirow{11}{*}{$\begin{array}{r}38.5 \% \text { strictly } \\
\text { anaerobe }\end{array}$} & \multicolumn{2}{|l|}{ Brain abscess (n=8) } & \multicolumn{2}{|l|}{ Subdural abscess $(n=5)$} & Epidural abscess (n=3) \\
\hline & Actinomyces meyeri & 1 & Escherichia coli & 1 & Staphylococcus aureus \\
\hline & Capnocytophaga spp & 1 & Staphylococcus aureus & 1 & \\
\hline & Cellulosimicrobium cellulans & 1 & Streptococcus intermedius & 2 & \\
\hline & Gemella morbillorum & 1 & Streptococcus pneumoniae & 1 & 1 \\
\hline & Parvimonas micra & 2 & & & $100 \%$ \\
\hline & Prevotella intermedia & $\begin{array}{l}1 \\
1\end{array}$ & & & $100 \%$ \\
\hline & Propionobacterium acnes & 2 & 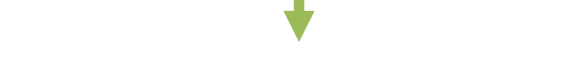 & & \\
\hline & Streptococcus constellatus & 1 & $40 \%$ & & \\
\hline & Streptococcus gordonii & 1 & & & \\
\hline & Streptococcus intermedius & 1 & & & \\
\hline
\end{tabular}

Treatment
Antibiotic therapy

- different empiric antibiotic treatment combinations

- Most frequent (43.8\%) ceftriaxone, vancomycin and metronidazole

- $15(93.8 \%)$ were submitted to surgery

- $56.3 \%$ received steroids

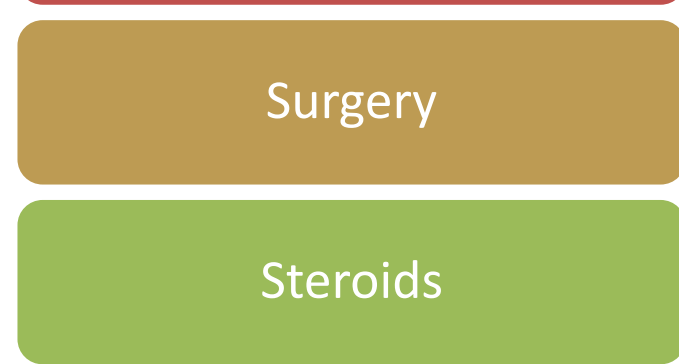

Outcome

- No deaths

- 2 cases of subdural abscesses, that were complications of other primary diagnoses, have sequelae

\section{CONCLUSIONS}

The frequent nonspecific clinical features at presentation might delay diagnosis and the lack of clear and consensual guidelines contribute to diverse therapeutic choices and treatment duration. MRl should be the imaging of choice. 